\title{
Mixed Electronic and Ionic Conduction Properties of Lithium Lanthanum Titanate
}

Michael J. Wang, Jeffrey B. Wolfenstine and Jeff Sakamoto*

M. Wang, Prof. J. Sakamoto

Department of Materials Science \& Engineering

University of Michigan

Ann Arbor, Ml 48109, USA

E-mail: jeffsaka@umich.edu

Prof. J. Sakamoto

Department of Mechanical Engineering

University of Michigan

Ann Arbor, MI 48109, USA

Dr. J. Wolfenstine

Solid lonic Consulting

Seattle, WA 98115

Keywords: batteries, electroceramics, electrolytes, conductivity, LLTO

With the continued increase in Li-metal anode rate capability, there is an equally important need to develop high-rate cathode architectures that are compatible with solid-state electrolytes. A proposed method of improving charge transport in the cathode is introducing a mixed electronic and ionic conductor (MEIC) which can eliminate the need for conductive additives that occlude electrolyte-electrode interfaces and lower the net additive required in the cathode. This study takes This is the author manuscript accepted for publication and has undergone full peer review but has not been through the copyediting, typesetting, pagination and proofreading process, which may lead to differences between this version and the Version of Record. Please cite this article as doi: 10.1002/adfm.201909140.

This article is protected by copyright. All rights reserved. 
advantage of a reduced perovskite electrolyte, $\mathrm{Li}_{0.33} \mathrm{La}_{0.57} \mathrm{TiO}_{3}$ (LLTO), to act as a model MEIC. It was found that the ionic conductivity of reduced LLTO is comparable to oxidized LLTO ( $\sigma_{\text {bulk }}=10^{-3}-10^{-4} \mathrm{~S}$ $\mathrm{cm}^{-1}, \sigma_{\mathrm{GB}}=10^{-5}-10^{-6} \mathrm{~S} \mathrm{~cm}^{-1}$ ) and the electronic conductivity is $1 \mathrm{mS} \mathrm{cm}^{-1}$. The ionic transference numbers were calculated to be 0.9995 and 0.0095 in the oxidized and reduced state respectively. Furthermore, two methods for controlling the transference numbers were evaluated. It was found that the electronic conductivity cannot easily be controlled by changing $\mathrm{O}_{2}$ overpressures, but increasing the ionic conductivity can be achieved by increasing grain size. This work identifies a possible class of MEIC materials that may improve rate capabilities of cathodes in solid-state architectures and motivate a deeper understanding of MEICs in the context of solid-state batteries.

\section{Introduction}

With the increasing need for vehicle electrification, there is a need to develop higher performance, safer, lower cost batteries ${ }^{[1,2]}$. For these reasons, solid-state batteries are emerging as a candidate to meet the energy density requirem ents for next generation electric vehicles. Due to their potential to enable Li metal anodes, significant effort has been made to study and stabilize the Li metal-solid electrolyte interface ${ }^{[3,4]}$. With recent progress in this area, studies have demonstrated stable cycling of Li in a variety of solid electrolytes, ranging from oxides ${ }^{[5-7]}$, to sulfides ${ }^{[8,9]}$, to polymers ${ }^{[10,11]}$, at current densities nearing the targets for electric vehicles $\left(>1 \mathrm{~mA} \mathrm{~cm}^{-2}\right)$. However, despite the significant progress in integrating Li metal with solid electrolytes, there has been a lack of emphasis on cathode integration. One of the major issues for cathodes in all solid-state batteries is the ease of ion and electron transport through the composite electrode construct ${ }^{[12,13]}$. Unlike conventional Li-ion batteries, there is noliquid electrolyte to infiltrate the cathode porosity and facilitate ion transport. Therefore, composite cathodes for all solid-state batteries require both ionconducting as well as electron-conducting additives to facilitate charge transport through the electrode thickness. However, with the inherently high resistance across solid-solid interfaces 
compared to solid-liquid interfaces, charge transport through composite cathodes remains a major hurdle to the development of fast-charging all solid-state batteries ${ }^{[12]}$.

Currently, cathodes for all solid-state batteries reported in the literature are prepared either by co-sintering or by coating. For co-sintered composite cathodes, the active material is cosintered with an ion-conducting additive, typically the same material as the solid-electrolyte, and electron-conducting additives like Ag or carbon black to create interpenetrating matrices of active material and conductive additive ${ }^{[9,14-16]}$. Alternatively, cathodes can be prepared similarly to conventional Li-ion cathodes but with an additional coating of Li-conducting additive ${ }^{[17-19]}$. Although these methods have shown some success, they are also limited by stability of the composite constituents, surface occlusion of active particles, and poor connectivity of conductive additive and active particles ${ }^{[12,13,17,18,20]}$. These strategies are illustrated in Figure 1. A potential solution for these issues which has been suggested ${ }^{[14]}$, is to develop mixed electronic and ionic conducting materials to either entirely replace or reduce the amount of different conductive additives, similarly to cathodes in solid-oxide fuel cells. By embedding the active particles within a matrix of a mixed-conducting phase, it diminishes the need for separate ion-conducting additive and electron-conducting additive. This may improve the connectivity of the active particles, enabling improved transport of ions toward and electrons away from the active particle surface. Furthermore, this introduces the potential to fabricate composite cathodes with high theoretical densities, which can improve the energy density and utilization.

In this study, lithium lanthanum titanium oxide (LLTO) was chosen as a model system that can exhibit mixed electronic and ionic conduction. LLTO perovskites have been widely studied as potential solid-state electrolytes, mainly due to their high bulk ionic conductivity $\left(1 \mathrm{mS} \mathrm{cm}^{-1}\right)$, which is the highest reported bulk conductivity for oxide materials ${ }^{[21,22]}$. However, one of the biggest limitations of LLTO is its instability against metallic Li, which results in immediate reduction of the 
$\mathrm{Ti}^{4+}$ to $\mathrm{Ti}^{3+}$, drastically increasing the electronic conductivity. As a solid electrolyte, a ny increase in the electronic conductivity short-circuits the cell. However, it may prove beneficial as a conductive additive for composite cathodes, as in its reduced state, LLTO becomes a mixed electronic and ionic conductor with relatively high conductivities.

In this work, $\mathrm{LLTO}$ of the composition $\mathrm{Li}_{0.33} \mathrm{La}_{0.57} \mathrm{TiO}_{3}$ was studied, comparing the differences in the physical and electrochemical properties in the oxidized (o-LLTO) and reduced ( $r$-LLTO) state. A combination of DC and AC electrochemical methods were performed to study the electronic and ionic conduction properties. Furthermore, two methods for controlling electronic and ionic conductivity of the reduced LLTO were evaluated, which may be necessary for application in composite cathodes. The first method aims to decrease the electronic conductivity by changing the $\mathrm{O}_{2}$ overpressure and the second method aims to increase the ionic conductivity by increasing the grain size. Similar to LLTO, there are several other materials that have been disregarded as solidelectrolytes because of dramaticincreases in electronic conductivity following spontaneous reduction of constituent elements (eg: $\mathrm{Ti}, \mathrm{Cr}, \mathrm{Nb}, \mathrm{Ge}, \mathrm{Bi}$, etc) as a result of reactions with the electrodes. This study focuses on LLTO as a model mixed electronic and ionic conducting system to study mixed conduction in the context of solid-state batteries and in the hopes to revisit other potential mixed conducting solid electrolyte materials.

\section{Experimental Results}

\subsection{Physical and Structural Properties}

It has been previously demonstrated that as-calcined LLTO powder, typically white in color, turns black, throughout the entire thickness of the densified material, when hot-pressed in a reducing atmosphere, indicating reduction of the $\mathrm{Ti}^{4+}$ to $\mathrm{Ti}^{3+}$ and a subsequent increase in the electronic conductivity ${ }^{[23-26]}$. In this study, this darkening phenomenon is confirmed as shown in 
Figure 2. Since the LLTO powder is hot-pressed in a graphite die and in Ar gas, there is a reduction of the $\mathrm{Ti}^{4+}$ to compensate for the loss of $\mathrm{O}_{2}$ from the structure. It was observed that the reduction of $\mathrm{Ti}^{4+}$ as indicated by the white-to-black color change only occurred at temperatures above $900^{\circ} \mathrm{C}$. After hot-pressing, the reduced LLTO ( $r$-LLTO) pellets exhibit no noticeable color change after days of sitting in ambient air at room temperature. In order to re-oxidize the r-LLTO, the samples were heattreated in air at $900^{\circ} \mathrm{C}$, as described by Wolfenstine et al. ${ }^{[23]}$. This heat-treatment successfully changed the color of the pellets from black to an ivory color throughout the thickness of the pellet, which is typical for oxidized LLTO (o-LLTO) in the literature. X-ray diffraction confirmed that there is negligible change in phase purity during this oxidation step. Figure 2 compares the visual color change for the as-hot-pressed sample and the oxidized sample as well as the XRD spectra for both samples. Furthermore, measurements of the physical dimensions confirmed that the relative densities of the pellets were $>95 \%$, which is typical for hot-pressed ceramics.

\subsection{Electrochemical Properties}

Electrochemical impedance spectroscopy (EIS) was conducted at room temperature on $r$ LLTO and o-LLTO samples using sputtered Au-electrodes. The EIS spectrum for o-LLTO is shown in Figure 3 and has a general shape that is consistent with EIS spectra for LLTO and other ceramic electrolytes with similar electrodes ${ }^{[23,27]}$. The Warburg element observed is indicative that the predominant physical process occurring at low frequencies is charge-transfer and semi-infinite diffusion of $\mathrm{Li}^{+}$in the $\mathrm{Au}^{[28]}$. As observed with other solid-electrolytes, there are two observable semi-circles, indicative of bulk conduction and grain boundary conduction, based on the calculated capacitances. In agreement with the literature, the resistance of the grain boundaries is orders of magnitude higher than the bulk ${ }^{[23,29,30]}$. The calculated conductivities are $0.73 \mathrm{mS} \mathrm{cm}^{-1}$ and $4.6 \times 10^{-6} \mathrm{~S}$ $\mathrm{cm}^{-1}$, for bulk and grain boundary respectively, which is consistent with other reports from the literature ranging from $0.5-1.0 \mathrm{mS} \mathrm{cm}^{-1[22,29-32]}$ and $3.0 \times 10^{-7} \mathrm{~S} \mathrm{~cm}^{-1}-7.5 \times 10^{-5} \mathrm{~S} \mathrm{~cm}^{-1[29-32]}$ respectively. 
This results in a total ionic conductivity of $1.6 \times 10^{-5} \mathrm{~S} \mathrm{~cm}^{-1}$. The EIS spectrum for $\mathrm{r}$-LLTO is also shown in Figure 3 and exhibits a drastically different shape than the o-LLTO spectrum. Unlike the o-LLTO spectra, no Warburg element is observed, and instead the low-frequency semi-circle fully intersects the $\operatorname{Re}(Z)$ axis. This is characteristic of a non-blocking configuration, which indicates that the dominant physical process occurring at low frequencies is electronic conduction rather than double layer capacitance from blocking behavior. This suggests that the $r$-LLTO is primarily acting as an electronic conductor, however, the presence of a semi-circle rather than a single point does indicate that the conductivity is insufficiently high to act as a pure metallic resistor.

Because the ionic current is mainly shunted by the electronic current in the case of $r$-LLTO, an electron blocking configuration was needed to measure the ionic conductivity. This method involves using an electrode material which is blocking to electrons while non-blocking to Li, thus acting as an electron filter. This method has been used for measuring the MEIC properties of other Li-ion materials like $\mathrm{LiFePO}_{4}{ }^{[33-35]}$. In this study, 1.0M solution-cast poly(ethylene oxide)-LiTFSI was used as the electron-blocking material with Li metal foils acting as a Li source and current collector. Figure $3 \mathrm{~b}$ shows the impedance spectra for the r-LLTO in the electron-blocking configuration. It can be seen that three semi-circles are observed, and based off of the calculated capacitances, these can be assigned to physical phenomena according to Irvine et al. ${ }^{[27]}$ (Table S1), with the high-frequency semi-circle representing the combination of bulk PEO and bulk LLTO conduction $\left(\sim 10^{-10}-10^{-11} \mathrm{~F}\right)$, a mid-frequency semi-circle representing LLTO grain-boundary conduction $\left(\sim 10^{-7}-10^{-8} \mathrm{~F}\right)$, and a lowfrequency semi-circle representing a combination of the interfacial resistance between Li/PEO and PEO/LLTO $\left(\sim 10^{-6} \mathrm{~F}\right)$. To measure the resistance of each component, the spectrum was fit to an equivalent circuit containing four RC units in series (Figure S1). In order to account for variations in the time constants, the capacitive element in each RC unit was replace d with a constant phase element (CPE). Given that the bulk conductivities for PEO are well defined, the bulk PEO and bulk This article is protected by copyright. All rights reserved. 
LLTO contributions can be deconvoluted within the circuit model, despite being within the same frequency regime. The calculated conductivities for the r-LLTO are $6.3 \times 10^{-4} \mathrm{~S} \mathrm{~cm}^{-1}$ and $9.1 \times 10^{-6} \mathrm{~S} \mathrm{~cm}^{-}$

${ }^{1}$ for bulk and grain boundary respectively. It can be seen that these values are consistent with the conductivities measured for the o-LLTO, suggesting that the reduction of the $\mathrm{Ti}^{4+}{ }^{\mathrm{i}}$ the lattice does not significantly affect the mobility of $\mathrm{Li}^{+}$.

Chronoamperometry was performed using sputtered-Au electrodes in order to measure the electronic conductivity. In this configuration, the initial transience in the current response is representative of both $\mathrm{Li}^{+}$and electron transport. However, since there is no supply of $\mathrm{Li}^{+}$from the electrodes, the supply of mobile ions is depleted and the residual current is purely electronic ${ }^{[36]}$. Figure 4 compares the current response for o-LLTO and r-LLTO. It is clear that the r-LLTO exhibits high electronic conductivity, five order of magnitudes higher than the o-LLTO. The resistances are calculated simply using Ohm's law at the steady state current values and used to calculate the electronic conductivities. The electronic conductivities for O-LLTO and r-LLTO are $2.4 \times 10^{-9} \mathrm{~S} / \mathrm{cm}$ and $9.5 \times 10^{-4} \mathrm{~S} / \mathrm{cm}$. In the case of $\mathrm{r}-\mathrm{LLTO}$, the electronic conductivity calculated from the DC measurements matches reasonably well to the resistance of the observed semi-circle in the AC impedance spectrum shown in Figure 2b. Slight deviations from resistive behavior are observed at higher potentials above 3.5V. The exact nature of these deviations is unknown but may be attributed to a potential dependence of the electron charge transfer resistance or higher degrees of Au lithiation and localized LLTO delithiation at higher potentials. If so, the actual electronic conductivities may be slightly lower than the reported values.

It was confirmed by Yang et al. ${ }^{[25]}$ and Wenzel et al. ${ }^{[26]}$ that the darkening phenomenon in LLTO is in fact due to the reduction of $\mathrm{Ti}^{4+}$ to $\mathrm{Ti}^{3+}$ within the crystal structure and is also accompanied by the dramatic increase in electronic conductivity. Using $x$-ray photoelectron spectroscopy (XPS), it was reported that reduction of about $12-24 \%$ of the $\mathrm{Ti}^{4+}$ in the lattice to $\mathrm{Ti}^{3+}$ was accompanied by an This article is protected by copyright. All rights reserved. 
increase in the electronic conductivity up to $\sim \mathrm{S} \mathrm{cm}^{-1}$. Given that the measured electronic conductivities in this work is about three orders of magnitude lower than these reported values, it is expected that the $\mathrm{Ti}^{3+}$ concentration is significantly lower than other reports in the literature.

Assuming that the electron carrier concentration is directly proportional to the concentration of $\mathrm{Ti}^{3+}$, it can be estimated that in this work, only about $0.1 \%$ of $\mathrm{Ti}^{4+}$ in the lattice has been reduced upon sintering, compared to the $12-24 \%$ reported for electrochemically reduced LLTO. XPS was performed on the samples and no noticeable differences between the r-LLTO and o-LLTO samples could be observed. This is in agreement with the estimated concentration of $\mathrm{Ti}^{3+}$ based off of Yang et al. and Wenzel et al. given that concentrations near $0.1 \%$ are not easily detectable using conventional XPS. This also suggests that a drastic darkening of the sample and subsequent increase in electronic conductivities (several orders of magnitude) can be achieved with relatively small concentrations of $\mathrm{Ti}^{3+}$.

\subsection{Transference Number}

From the combination of AC and DC measurements, it can be seen that o-LLTO is primarily an ion-conductor with an ionic transference number of 0.9995 while $r$-LLTO is primarily an electronconductor with an electronic transference number of 0.9905. For a mixed conductor at steady state, local charge neutrality dictates that for all charged species $i$ :

$\sum_{i} z_{i} J_{i}=0$

where $z$ is the valence, and $J$ is the charge flux density. The flux density can be written in a form similar to Fick's first law as:

$J_{i}=-\widetilde{D}_{\mathrm{i}} \nabla c_{i}$

This article is protected by copyright. All rights reserved. 
Where $\widetilde{D}$ is defined by Weppner \& Huggins as the chemical diffusion coefficient (effective diffusion coefficient $)^{[37]}$, which is related to the diffusivity by an enhancement factor $\mathrm{W}$ :

$\widetilde{D}_{\mathrm{i}}=D_{i} W=D_{i}\left[\left(1-t_{i}\right) \frac{\partial \log \left(a_{i}\right)}{\partial \log \left(c_{i}\right)}-\sum_{j \neq i} t_{j} \frac{z_{i}}{z_{j}} \frac{\partial \log \left(a_{j}\right)}{\partial \log \left(c_{j}\right)}\right]$

where $\mathrm{W}$ is the enhancement factor, $\mathrm{D}$ is the diffusivity, a is the activity, $\mathrm{c}$ is the concentration, and $\mathrm{t}$ is the transference number. The transference number is defined by the conductivity $\sigma$ of each species:

$t_{i}=\frac{\sigma_{\mathrm{i}}}{\sum_{j} \sigma_{\mathrm{j}}}$

where $\sigma$ is the conductivity. For MEICs in composite cathodes, it would be ideal for the ionic current and electronic current to contribute equally such that the rate of incoming/outgo ing $\mathrm{Li}^{+}$is comparable to the rate of outgoing/incoming $\mathrm{e}^{-[37,38]}$. Because the currents under an applied potential are a function of the transference numbers, this is represented by the relationship:

$J_{e^{-}}\left(t_{e^{-}, t_{L i^{+}}}\right)=J_{L i^{+}}\left(t_{e^{-}, t_{L i^{+}}}\right)$

Therefore, the ability to control the ionic/electronic conductivities may be necessary to meet this condition and design viable composite cathodes. In the following section, two potential methods of controlling the transference number of r-LLTO are suggested. The first method proposes a decrease in the electronic conductivity by controlling the Ti valence state and the second method proposes increasing the ionic conductivity by increasing the grain size.

\subsection{Electrochemical Response to Changes in $p_{O 2}$}

As the high electronic conductivity in r-LLTO is due to the reduction of $\mathrm{Ti}^{4+}$ to $\mathrm{Ti}^{3+}$, a potential way to decrease the electronic conductivity may be to partially re-oxidizethe $\mathrm{Ti}^{3+}$ ions. In this study, LLTO samples were fabricated in the r-LLTO state and then annealed in $\mathrm{O}_{2}$ to attain the o-LLTO state.

This article is protected by copyright. All rights reserved. 
Presumably, the increase in $\mathrm{O}_{2}$ overpressure decreases the concentration of oxygen vacancies, requiring the $\mathrm{Ti}^{3+}$ to re-oxidize to $\mathrm{Ti}^{4+}$ to maintain charge neutrality. Assuming the initial valence of the $\mathrm{Ti}$ in the lattice is $\mathrm{Ti}^{3+}$, this is represented (using Kroger-Vink notation) by the defect relations:

$$
\begin{aligned}
& O_{O}^{X}=\frac{1}{2} O_{2}(g)+V_{O} \ddot{ }+2 e^{\prime} \\
& {\left[e^{\prime}\right]=\left[T i_{T i}^{\prime}\right]} \\
& K=\frac{p_{O 2}^{\frac{1}{2}}\left[V_{O}^{\prime \prime}\right]\left[e^{\prime}\right]^{2}}{\left[O_{O}^{X}\right]}
\end{aligned}
$$

where $K$ is the equilibrium constant and $p_{O_{2}}$ is the partial pressure of oxygen. Based on the charge neutrality equation the electron concentration $\left[e^{\prime}\right]$ can be expressed as:

Charge neutrality: $\left[e^{\prime}\right]=2\left[V_{o}^{*}\right]$

$K=p_{O 2}^{\frac{1}{2}}\left[\frac{e^{\prime}}{2}\right]\left[e^{\prime}\right]^{2}$

$K=\exp \left(-\frac{\Delta G}{k T}\right)$

$\left[e^{\prime}\right] \approx p_{O 2}^{-\frac{1}{6}} \exp \left(-\frac{\Delta G}{3 k T}\right)=f\left(p_{O 2}, T\right)$

From this equation it can be seen that the electron concentration and therefore the electronic conductivity is a function of the $p_{O 2}$ and the temperature. As the $p_{O 2}$ increases, it is expected that the concentration of $\mathrm{Ti}^{3+}$ decreases and therefore a decrease in electronic conductivity should occur. Furthermore, the proposed defect reaction which asserts that the change in Ti valence is the primary means to maintain charge neutrality, rather than a change in Li concentration supports the lack of change in the $\mathrm{Li}^{+}$conductivity in the oxidized and reduced states. Therefore, the ability to transition between the r-LLTO and o-LLTO states suggests that intermediate values for the electronic

This article is protected by copyright. All rights reserved. 
conductivity may be achieved without compositional changes, simply by altering the concentrations of $\mathrm{Ti}^{4+}$ and $\mathrm{Ti}^{3+}$ by means of the temperature and $\mathrm{O}_{2}$ overpressure. To evaluate the ability to control the electronic conductivity of the LLTO samples, the electronic conductivities were measured in situ at varying temperatures and atmospheres and is depicted in Figure 5. In general, it was observed that at elevated temperatures and high $p_{02}$, the reduced samples favored a completely oxidized state, as indicated by drastic drops in electronic conductivity. $\ln p_{O 2}=1.0$, the $r$-LLTO samples spontaneously oxidize at ambient temperatures, which is indicated by the low electronic conductivity values, as previously calculated. For atmospheres with $p_{O 2}=0.5$ and $p_{O 2}=0.75$, the transition temperature is $150^{\circ} \mathrm{C}$, while at $p_{O 2}=0.25$ the transition temperature is $300^{\circ} \mathrm{C}$. Furthermore, in $p_{O 2}=10^{-7}(\mathrm{Ar})$, the samples oxidize at $900^{\circ} \mathrm{C}$. Given, the typical diffusion coefficients for $\mathrm{O}$ in ion conducting oxides, the system is unlikely to be at its equilibrium state at each temperature and would likely take more than several days to equilibrate at each temperature. Despite this, it can be seen that the dramatic decrease in electroni c conductivity still occurs within a relatively short period of time in comparison to the time necessary to fully reach equilibrium. These results imply that the transition from the reduced to oxidized state happens rather abruptly, as illustrated by the sudden discontinuity in the conductivity as a function of temperature for the $p_{O 2}$ sample in Figure 5. This may be due to the low concentration ( $0.1 \%)$ of $\mathrm{Ti}^{3+}$ in the r-LLTO samples as previously estimated.

Figure 5 also shows the Arrhenius behavior of the electronic conductivity in the $p_{O 2}=1$. and 0.25 environments. As expected, there is a linear relationship between the $\ln (\sigma)$ and $T^{1}$, however, a discontinuity at $450^{\circ} \mathrm{C}$ indicates that there are two distinct mechanisms which govern electron conduction. In the low temperature region, electronic conductivity likely originates from a polaron hopping mechanism ${ }^{[39]}$. In this case the electron concentration $\left[e^{\prime}\right]$ is given by Equation 12 . From Equation 12 it is observed that at a given temperature $\left[e^{\prime}\right]$ is determined by the $p_{02}$, as the $p_{02}$ This article is protected by copyright. All rights reserved. 
increases the $[e]$ decreases. With the assumption that electron mobility is independent of $p_{O 2}{ }^{[39]}$ the electronic conductivity should vary with $p_{O 2}$ in the same way the $[e]$ varies with $p_{O 2 .}$. From Figure 5 it is observed that as $p_{O 2}$ increases in the low temperature region electronic conductivity decreases, in agreement with Equation 12. Furthermore, in the low temperature region if polaron conductivity is the dominant mechanism, the activation energy for electronic conductivity (assuming $p_{O 2}$ independent mobility) given by Equation 12 should be the same for both the oxidized and reduced samples, the only difference being the $[e]$ which is controlled by the $p_{O 2}$. The activation for the reduced and oxidized samples in the low temperature is $0.06 \mathrm{eV}$ and $0.08 \mathrm{eV}$, respectively. The similar values suggest that the low temperature region of both the oxidized and reduced samples is controlled by the same mechanism, most likely polaron hopping. At a given temperature the difference in electronic conductivity between the reduced and oxidized samples is due only to the effect of $p_{O 2}$ on the $\left[e^{\prime}\right]$.

Above $450^{\circ} \mathrm{C}$ the electronic conductivity of the reduced and oxidized samples at the different $p_{O 2}$ fall on the same curve. Furthermore, above $450^{\circ} \mathrm{C}$ an increase in activation energy $(0.35 \mathrm{eV})$ compared to the low temperature region $(0.08 \mathrm{eV})$ is obsened. This would suggest a new conductivity mechanism, other than polaron hopping, which is independent of $p_{O 2 .}$. It could be due to impurities but they usually dominate at temperatures below those where polarons control electronic conductivity ${ }^{[39]}$. Thus, the new region above $450^{\circ} \mathrm{C}$ is not due to impurities. It is likely that in this region the electronic conductivity is a result of electronic transition across the band gap. In this case the activation energy for crossing the bad gap would be higher than that for polaron conductivity ${ }^{[39,40]}$ and hence, would control electronic conductivity at high temperatures which is in agreement with results of Figure 5. Furthermore, if the electronic conductivity is controlled by crossing the band gap, the electronic conductivity would be independent of $p_{O 2}$ and be the same for both the high and low $p_{\mathrm{O}_{2}}$ samples. This is in agreement with results of Figure 5, where it is observed

This article is protected by copyright. All rights reserved. 
that in the high temperature the electronic conductivity is independent of $p_{\mathrm{O} 2}$ for both samples and they have the same activation energy in this region. At low temperatures polaron conductivity dominates in which case, electronic conductivity is a function of $p_{O 2}$. At higher temperature it is suggested that electronic conductivity is controlled by the band gap, leading to a $p_{O 2}$ independent conductivity.

It is evident in Figure 5 that the transition from r-LLTO to o-LLTO is discrete rather than continuous, which was also observed for all $p_{O 2}$ between $p_{O 2}=0.25$ and $p_{O 2}=1$. This suggests that it may be difficult to achieve intermediate values of the electronic conductivity using this approach. This would mean that it may not be viable using heat treatments to finely control the electronic conductivity for achieving ideal transference numbers. The LLTO will only be able to exhibit low conductivity or high conductivity and not be able to exhibit intermediate values. Although it may be possible in theory, the inability to precisely control small quantities of $\mathrm{Ti}^{3+}(<0.1 \%)$ using heat treatments alone may be the primary limiting factor. For this reason, partial reduction of o-LLTO was not explored in this work. In this case, other approaches to finely control the electronic conductivity may prove to be a more viable strategy. One possible method to achieve a mixed valence state of the Ti atoms may be by aliovalent doping. Wolfenstine et al. demonstrated the ability to control the electron concentration and therefore the electronic conductivity in $\mathrm{Li}_{4} \mathrm{Ti}_{5} \mathrm{O}_{12}$ via Ta doping ${ }^{[41]}$. Alternatively, methods that can maintain atmospheres ( $p_{o 2}$ and temperature) with high levels of precision during sintering or post-sintering may be able to better control the mixed valence state. However, solely using simple heat treatment approaches as demonstrated, the electronic conductivity is a non-trivial parameter to finely control and therefore may not be a viable method of tuning the transference number.

This article is protected by copyright. All rights reserved. 


\subsection{Effect of Grain Size on Conductivity}

Rather than decrease the electronic conductivity, another approach to control the transference number is to increase the ionic conductivity. Since it is well understood that the ionic conductivity of LLTO is limited by the poor grain boundary conduction ${ }^{[42]}$, one strategy to improve the ionic conductivity is to increase the average grain size. As expected, thi s has been shown to be the case by Ban and $\mathrm{Choi}^{[29,43]}$. In order to increase the average grain size, the samples were annealed in Arfor $10 \mathrm{hrs}, 20 \mathrm{hrs}$, and $50 \mathrm{hrs}$. Visual inspection as well as XRD confirm that the samples remain in the reduced state with negligible change in phase purity after annealing. Figure 6 shows electron backscatter diffraction (EBSD) maps for the as-hot-pressed and the annealed samples. The average grain size for each is $0.8 \mu \mathrm{m}, 1 \mu \mathrm{m}, 2 \mu \mathrm{m}$, and $10 \mu \mathrm{m}$. By fitting the grain size as a function of annealing time to the equation:

$d^{n}-d_{0}^{n}=A(T) t$

where $d$ is the grain size, $d_{0}$ is the original grain size, $A$ is a temperature-dependent constant, $t$ is the annealing time, and $n$ is the grain-growth exponent, it was determined that a grain-growth exponent of $n=2$ resulted in the best fit, with an $R^{2}$ value of 0.95 . This is indicative that the grain growth in LLTO is representative of normal grain growth, which is not limited by secondary phases or pores ${ }^{[44,45]}$. EIS and chronoamperometry were performed on each sample using the previously mentioned configurations. It was observed that there is a roughly linear increase in the ionic grain boundary conductance with increasing grain size, which is consistent with the observations of Ban and $\mathrm{Choi}^{[29]}$. As expected, a one order of magnitude increase in the grain size ( $1 \mu \mathrm{m}$ to $\left.10 \mu \mathrm{m}\right)$ decreases the grain boundary resistance by one order of magnitude $(1 \mathrm{M} \Omega \mathrm{cm}$ to $0.1 \mathrm{M} \Omega \mathrm{cm})$. The magnitude of change is also in good agreement with the findings of Ban and Choi ${ }^{[29]}$. While the ionic grain boundary resistance decreases with increasing grain size, the DC methods show that the electronic conductivity does not significantly change with grain size. This suggests that the high

This article is protected by copyright. All rights reserved. 
electronic conductivity of $r$-LLTO is not significantly impeded by grain boundaries. The relatively small mean free paths ( $1-100 \mathrm{~nm})$ of the electrons compared to the grain size $(>1 \mu \mathrm{m})$ may explain the independence of electronic conductivity from grain size ${ }^{[46,47]}$. In terms of controlling the transference number, it would seem that increasing the ionic conductivity may be an easier method than decreasing the electronic conductivity. Although the demonstrated relationship is shown to be linear, it is expected that the trend should eventually asymptote toward a grain size-independent value at large grain sizes ${ }^{[48]}$. Therefore, in order to achieve ionic conductivities comparable to the electronic conductivities, significantly larger grains ( $>100 \mu \mathrm{m}$ based on the observed trends) or near single grains will be required. Alternatively, promising results in terms of ionic conductivity have been demonstrated in amorphous LLTO, which may be a promising solution for achieving comparable ionic and electronic conductivities ${ }^{[49]}$.

\section{Implications for Solid-State Batteries}

LLTO was first considered as a promising solid-electrolyte due to its high ionic conductivity. However, the most important criterion for any battery electrolyte is a low electronic conductivity to prevent self-discharge. This is the primary reason why o-LLTO is a viable solid-state electrolyte but becomes a poor electrolyte upon spontaneous reduction from Li metal contact. Although, o-LLTO may be compatible against other less reducing electrodes, it is unlikely to enable Li metal anodes which are the biggest motivation for developing solid-state batteries. However, in a composite cathode configuration, a mixed conductor, like r-LLTO, may eliminate the need for separate ionic and electronic conductive additives. Rather than having two percolative networks of conductive additives which lowers the fraction of active material and may suffer from poor connectivity, a single percolative network of mixed conducting material may allow for higher active loadings and more facile ionic/electronic transport toward/away from the active particles. It is demonstrated here that mixed conduction of $\mathrm{Li}^{+}$and electrons can be achieved in a relatively well-studied system, which may

This article is protected by copyright. All rights reserved. 
suggest the possibility of revisiting other electrolytes that undergo spontaneous reduction, such as $\mathrm{Li}_{6.5} \mathrm{La}_{3} \mathrm{Zr}_{1.5} \mathrm{Nb}_{0.5} \mathrm{O}_{12}{ }^{[50,51]}$ or $\mathrm{Li}_{10} \mathrm{GeP}_{2} \mathrm{~S}_{12}{ }^{[52,53]}$. However, in order to achieve transport of both $\mathrm{Li}^{+}$and electrons at rates relevant to Li-ion batteries, it will be necessary for the total conductivities of both species to be on the order of $\sim 10^{-4}-10^{-3} \mathrm{~S} \mathrm{~cm}^{-1}$, ideally with similar transference numbers for reasons previously discussed. Simultaneously satisfying all of these requirements poses a significant challenge given the issues of transport across solid-solid interfaces, electrochemical stability, and fabrication, but may provide a novel solution to improving the rate capability of cathodes in all -solidstate architectures.

\section{Conclusion}

With the improving capabilities to cycle Li metal with solid state electrolytes at high current densities, there is a growing need to improve the rate capabilities of compatible cathodes. To date, the concept of using MEIC materials in the context of solid-state batteries has not been extensively explored. The goal of this study was to investigate the electrochemical properties of a known MEIC material, which has often been disregarded as a solid-state battery material because of those very properties. It was demonstrated here that $r$-LLTO can be readily synthesized by densifying in a reducing environment and shows notable physical and electrochemical properties from its o-LLTO counterpart. Unlike o-LLTO, which has a measured ionic transference number of 0.9995, r-LLTO was measured to have an electronic transference number of 0.9905 , which is due to the five order of magnitude increase in the electronic conductivity upon reduction. In the context of composite cathode configurations, it may be necessary for the transference number to be less skewed toward a particular charged species and therefore the effects of $p_{\mathrm{O}_{2}}$ and grain size on transference number were evaluated. While increasing the grain size of the r-LLTO improves the ionic conductivity, the electronicconductivity could not easilybe controlled by changing the $p_{O 2}$ in the atmosphere.

Overall, this work investigated the MEIC properties of a common solid electrolyte material, LLTO,

This article is protected by copyright. All rights reserved. 
which may give insight on the designs of composite cathode for solid state batteries and motivate further study of other potential MEIC materials for this application.

\section{Experimental Section}

Material Synthesis:

Lithium lanthanum titanate powder of the composition $\mathrm{Li}_{0.33} \mathrm{La}_{0.57} \mathrm{TiO}_{3}$ was purchased from NEI Corporation (Somerset, NJ) and densified using a rapid induction hot-pressing method. This composition of LLTO was used specifically as it is known to have the highest bulkionic conductivity ${ }^{[22]}$. The LLTO powder was pressed in a graphite die under a load of 40 MPa while heated at a temperature of $1050^{\circ} \mathrm{C}$. During the entirety of the hot-pressing process, the graphite die is contained within a stream of Ar flow, creating a reducing environment. It is known that ordering of the La atoms at high temperatures within the lattice can result in a tetragonal distortion ${ }^{[22,54]}$. Therefore, in order to achieve cubic LLTO without modifying the composition, the LLTO is cooled at a relatively rapid rate of $\sim 35^{\circ} \mathrm{C} \mathrm{min}^{-1}$, which should be sufficiently fast to achieve primarily cubic LLTO, despite the presence of tetragonal phase LLTO in the as-received powder ${ }^{[55]}$. The resulting billets were then cut into pellets with a diamond saw and polished with a variety of sandpapers and diamond pastes, beginning at a 1200 grit sandpaper and ending with a final polish using a $0.5 \mu \mathrm{m}$ diamond paste and a mineral-oil based polishing fluid (Leco, St. Joseph, MI). As reported in the literature, after hot-pressing, the white-colored LLTO powder turns black after hot-pressing, indicating a reduction of the $\mathrm{Ti}^{4+}$ to $\mathrm{Ti}^{3+[23]}$. For the oxidized samples, the hot-pressed pellets were heat-treated in air at $900^{\circ} \mathrm{C}$ for $2 \mathrm{hrs}$ and covered in mother powder to reduce the amount of $\mathrm{Li}$ sublimation. Samples were stored in an Ar-filled glovebox immediately after processing.

This article is protected by copyright. All rights reserved. 


\section{Material Characterization:}

X-ray diffraction (XRD) was conducted using Cu Ka radiation using a Rigaku Miniflex 600x-ray diffractometer to determine phase purity. The samples were scanned from 15 to 65 degrees with a 0.02 degree step size. In order to investigate the effect of grain size on the mixed conductivity of the reduced LLTO, the hot-pressed samples were heated for various times at elevated temperature to increase the grain size. The individual pellets were covered in mother powder to reduce Li loss and contained in a tightly sealed molybdenum foil packet to minimize exposure to atmosphere. The samples were heated in a Sentro Tech STT-1700C-2.5-12 tube furnace (Strongsville, $\mathrm{OH}$ ) at $1150^{\circ} \mathrm{C}$ for times of 10,20 and $50 \mathrm{hrs}$ under a constant $2 \mathrm{~L} \mathrm{~min}^{-1}$ flow of $\mathrm{Ar}$, to prevent oxidation of the sample. The grain size was determined using a Tescan MIRA3 GMU FEG scanning electron microscope equipped with electron backscatter diffraction (EBSD). For EBSD analysis, the microscope was operated at an accelerating voltage of $20 \mathrm{kV}$, a working distance of $25 \mathrm{~mm}$, and a tilt angle of $70^{\circ}$. Data analysis was performed using EDAX OIM software. In order to eliminate indexing noise for sub-micron noise, the datasets were refined under the assumption that only pixel groupings larger than $0.75 \mu \mathrm{m}$ are representative of grains.

\section{Electrochemical Characterization:}

The conductivities of the samples were measured using a combination of $A C$ and DC methods using a Bio-logic VMP300 galvanostat/potentiostat. Electrochemical impedance spectroscopy (EIS) was conducted to measure the ionic conductivity, using two configurations of electrodes. The first configuration consisted of two sputtered Au electrodes, acting as Warburg elements limited by Li diffusion in Au at low frequencies. The second configuration consisted of two thin films $(150 \mu \mathrm{m})$ of 1.0M poly(ethylene oxide)-LiTFSI (PEO-LiTFSI) which is blocking to electron transport but non-blocking to $\mathrm{Li}^{+}$transport at low frequencies. The PEO-LiTFSI films were 
synthesized by solution casting, as described by Gupta et al. ${ }^{[56]}$. The PEO-LiTFSI films were pressed against the polished LLTO pellets and then $200 \mu \mathrm{m}$ Li foils (Alfa Aesar) were pressed against the opposite side of the PEO films to act as both a current collector and Li source. The Li-PEO-LLTO-PEOLi stack was heated under slight pressure at $80^{\circ} \mathrm{C}$ to improve contact between each component. The EIS was conducted using a $10 \mathrm{mV}$ perturbation voltage at frequencies ranging from $500 \mathrm{mHz}$ to $7 \mathrm{MHz}$. The electronic conductivities were measured with sputtered Au electrodes using staircase potentiometry (Mott-Schottky), with voltage steps of $1.0 \mathrm{~V}$, from $0.5 \mathrm{~V}$ to $5.5 \mathrm{~V}$ vs the open circuit potential. EIS measurements were performed at the end of each voltage step using the same parameters as the EIS measurements performed without an applied potential.

High-temperature conductivity measurements were also conducted using chronoamperometry. The samples were coated with Au paste electrodes and held in a custom -built sample holder in a tube furnace under a flowing mixture of $\mathrm{Ar}_{\text {and }} \mathrm{O}_{2}$ gas. The partial pressure of $\mathrm{O}_{2}$ was controlled by adjusting the flow rate and inlet pressure of the two gases and the samples were held at increasing temperatures ranging from $150^{\circ} \mathrm{C}$ to $900^{\circ} \mathrm{C}$. The samples were held for $2 \mathrm{hrs}$ at each temperature before chronoamperometry was performed with a potential of $1 \mathrm{~V}$ vs the opencircuit potential.

\section{Supporting Information}

Supporting Information is available from the Wiley Online Library or from the author.

\section{Acknowledgements}

This work was supported by the Ford Motor Company Alliance Program. The authors would like to thank E. Kazyak and N. Dasgupta for conducting XPS measurements.

This article is protected by copyright. All rights reserved. 
Received: ((will be filled in by the editorial staff))

Revised: ((will be filled in by the editorial staff)) Published online: ((will be filled in by the editorial staff))

\section{References}

[1] J.-M. Tarascon, M. Armand, Nature 2001, 414, 359.

[2] B. D. McCloskey, J. Phys. Chem. Lett. 2015, 6, 3592.

[3] J. A. Lewis, J. Tippens, F. J. Q. Cortes, M. T. McDowell, Trends in Chemistry 2019, S2589597419301595.

[4] P. Albertus, S. Babinec, S. Litzelman, A. Newman, Nature Energy 2018, 3, 16.

[5] N. J. Taylor, S. Stangeland-Molo, C. G. Haslam, A. Sharafi, T. Thompson, M. Wang, R. GarciaMendez, J. Sakamoto, Journal of Power Sources 2018, 396, 314.

[6] C.-L. Tsai, V. Roddatis, C. V. Chandran, Q. Ma, S. Uhlenbruck, M. Bram, P. Heitjans, O. Guillon, ACS App/Mater Interfaces 2016, 8, 10617.

[7] R. H. Basappa, T. Ito, H. Yamada, Journal of The Electrochemical Society 2017, 164, A666.

[8] Y. Kato, S. Hori, T. Saito, K. Suzuki, M. Hirayama, A. Mitsui, M. Yonemura, H. Iba, R. Kanno, Nature Energy 2016, 1, 16030.

[9] M. Tatsumisago, M. Nagao, A. Hayashi, Journal of Asian Ceramic Societies 2013, 1, 17.

[10] Q. Lu, Y.-B. He, Q. Yu, B. Li, Y. V. Kaneti, Y. Yao, F. Kang, Q.-H. Yang, Advanced Materials 2017, 29,1604460 .

[11] M. Zhang, A. L. Gui, W. Sun, J. Becking, O. Riedel, X. He, D. Berghus, V. Siozios, D. Zhou, T. Placke, M. Winter, P. Bieker, J. Electrochem. Soc. 2019, 166, A2142.

[12] K. Kerman, A. Luntz, V. Viswanathan, Y.-M. Chiang, Z. Chen, J. Electrochem. Soc. 2017, 164, A1731.

[13] A. Bielefeld, D. A. Weber, J. Janek, J. Phys. Chem. C 2019, 123, 1626.

[14] A. J. Samson, K. Hofstetter, E. Wachsman, V. Thangadurai, Journal of The Electrochemical Society 2018, 165, A2303.

[15] S. Ohta, J. Seki, Y. Yagi, Y. Kihira, T. Tani, T. Asaoka, Journal of Power Sources 2014, 265, 40. 
[16] S. Ohta, S. Komagata, J. Seki, T. Saeki, S. Morishita, T. Asaoka, Journal of Power Sources 2013, 238, 53.

[17] N. Ohta, K. Takada, I. Sakaguchi, L. Zhang, R. Ma, K. Fukuda, M. Osada, T. Sasaki, Electrochemistry Communications 2007, 9, 1486.

[18] S. Noh, W. T. Nichols, C. Park, D. Shin, Ceramics International 2017, 43, 15952.

[19] C.-L. Xu, W. Xiang, Z.-G. Wu, Y.-C. Li, Y.-D. Xu, W.-B. Hua, X.-D. Guo, X.-B. Zhang, B.-H. Zhong, Journal of Alloys and Compounds 2018, 740, 428.

[20] S.-W. Baek, J.-M. Lee, T. Y. Kim, M.-S. Song, Y. Park, Journal of Power Sources 2014, 249, 197.

[21] O. Bohnke, Solid State Ionics 2008, 179, 9.

[22] S. Stramare, V. Thangadurai, W. Weppner, Chemistry of Materials 2003, 15, 3974.

[23] J. Wolfenstine, J. L. Allen, J. Read, J. Sakamoto, G. Gonalez-Doncel, Journal of Power Sources 2010, 195, 4124.

[24] Y. J. Shan, L. Chen, Y. Inaguma, M. Itoh, T. Nakamura, Journal of Power Sources 1995, 54, 397.

[25] K.-Y. Yang, I.-C. Leu, K.-Z. Fung, M.-H. Hon, M.-C. Hsu, Y.-J. Hsiao, M.-C. Wang, J. Mater. Res. 2008, 23, 1813.

[26] S. Wenzel, T. Leichtweiss, D. Krüger, J. Sann, J. Janek, Solid State lonics 2015, 278, 98.

[27] J. T. S. Irvine, D. C. Sinclair, A. R. West, Adv. Mater. 1990, 2, 132.

[28] C. Ho, I. D. Raistrick, R. A. Huggins, J. Electrochem. Soc. 1980, 127, 343.

[29] C. Ban, Solid State Ionics 2001, 140, 285.

[30] Y. Inaguma, C. Liquan, M. Itoh, T. Nakamura, T. Uchida, H. Ikuta, M. Wakihara, Solid State Communications 1993, 86, 689.

[31] A. Mei, Q.-H. Jiang, Y.-H. Lin, C.-W. Nan, Journal of Alloys and Compounds 2009, 486, 871.

[32] J.-G. Kim, H.-G. Kim, H.-T. Chung, Journal of Materials Science Letters 1999, 18, 493.

[33] R. Amin, J. Maier, P. Balaya, D. P. Chen, C. T. Lin, Solid State lonics 2008, 179, 1683.

[34] C. Wang, J. Hong, Electrochemical and Solid-State Letters 2007, 10, A65.

[35] R. Amin, Y.-M. Chiang, Journal of The Electrochemical Society 2016, 163, A1512.

[36] R. A. Huggins, lonics 2002, 8, 300.

[37] W. Weppner, R. A. Huggins, J. Electrochem. Soc. 1977, 124, 1569.

This article is protected by copyright. All rights reserved. 
[38] I. Riess, Solid State Ionics 2003, 157, 1.

[39] W. D. Kingery, H. K. Bowen, D. R. UhImann, Introduction to Ceramics, Wiley, New York, 1976.

[40] W. Weppner, Journal of Solid State Chemistry 1977, 20, 305.

[41] J. Wolfenstine, J. L. Allen, Journal of Power Sources 2008, 180, 582.

[42] C. Ma, K. Chen, C. Liang, C.-W. Nan, R. Ishikawa, K. More, M. Chi, Energy \& Environmental Science 2014, 7, 1638.

[43] A.C. Sutorik, J. Ceram. Sci. Tech. 2013, DOI 10.4416/JCST2012-00035.

[44] D. Fan, S. P. Chen, L.-Q. Chen, J. Mater. Res. 1999, 14, 1113.

[45] B.-N. Kim, K. Hiraga, K. Morita, n.d., 6.

[46] S. Kasap, C. Koughia, H. E. Ruda, in Springer Handbook of Electronic and Photonic Materials (Eds.: S. Kasap, P. Capper), Springer International Publishing, Cham, 2017, pp. 1-1.

[47] A. Tschöpe, Solid State lonics 2001, 139, 267.

[48] A. C. Sutorik, M. D. Green, C. Cooper, J. Wolfenstine, G. Gilde, J Mater Sci 2012, 47, 6992.

[49] S. Furusawa, H. Tabuchi, T. Sugiyama, S. Tao, J. Irvine, Solid State lonics 2005, 176, 553.

[50] Y. Kim, A. Yoo, R. Schmidt, A. Sharafi, H. Lee, J. Wolfenstine, J. Sakamoto, Front. Energy Res. 2016, 4, DOI 10.3389/fenrg.2016.00020.

[51] H. Nemori, Y. Matsuda, S. Mitsuoka, M. Matsui, O. Yamamoto, Y. Takeda, N. Imanishi, Solid State lonics 2015, 282, 7.

[52] F. Han, Y. Zhu, X. He, Y. Mo, C. Wang, Advanced Energy Materials 2016, 6, 1501590.

[53] S. P. Ong, Y. Mo, W. Davidson Richards, L. Miara, H. Sug Lee, G. Ceder, Energy \& Environmental Science 2013, 6, 148.

[54] O. Bohnke, H. Duroy, J.-L. Fourquet, S. Ronchetti, D. Mazza, Solid State lonics 2002, 10.

[55] X. Hu, X. Cheng, S. Qin, G. Yan, J. Malzbender, W. Qiang, B. Huang, Ceramics International 2018, 44, 1902.

[56] A. Gupta, E. Kazyak, N. Craig, J. Christensen, N. P. Dasgupta, J. Sakamoto, Journal of The Electrochemical Society 2018, 165, A2801.

This article is protected by copyright. All rights reserved. 


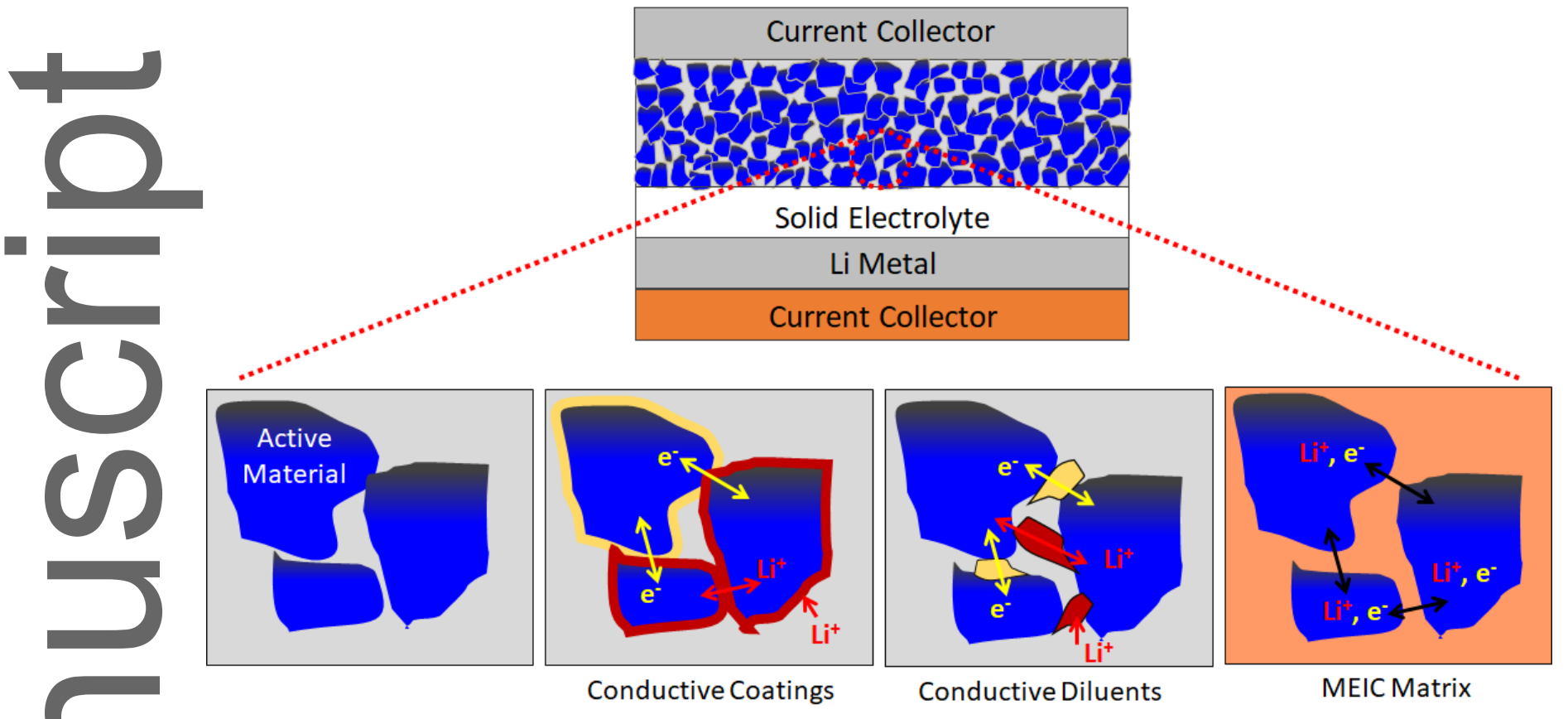

Figure 1. Schematic of a composite cathode within an all-solid-state-battery. Different strategies for improving the transport of $\mathrm{Li}^{+}$and $\mathrm{e}^{-}$between cathode particles are illustrated.

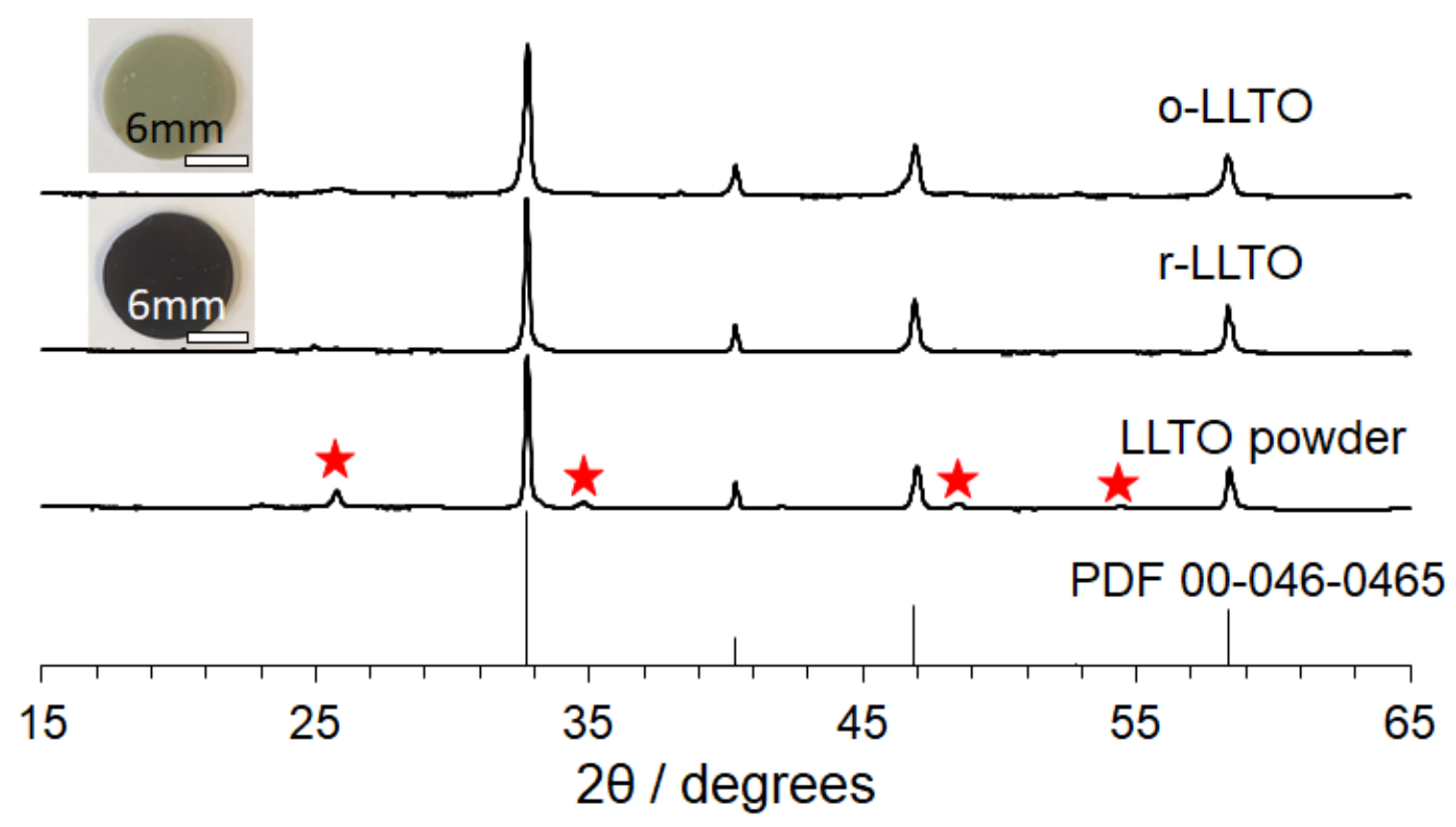

This article is protected by copyright. All rights reserved. 
Figure 2. XRD spectra for the calcined powder, r-LLTO, and o-LLTO. A reference for cubic perovskite LLTO is provided, PDF 00-046-0465. Stars indicate peaks belonging to the tetragonal perovskite phase. Visual comparison between as hot-pressed r-LLTO and oxidized o-LLTO is also shown.

a)

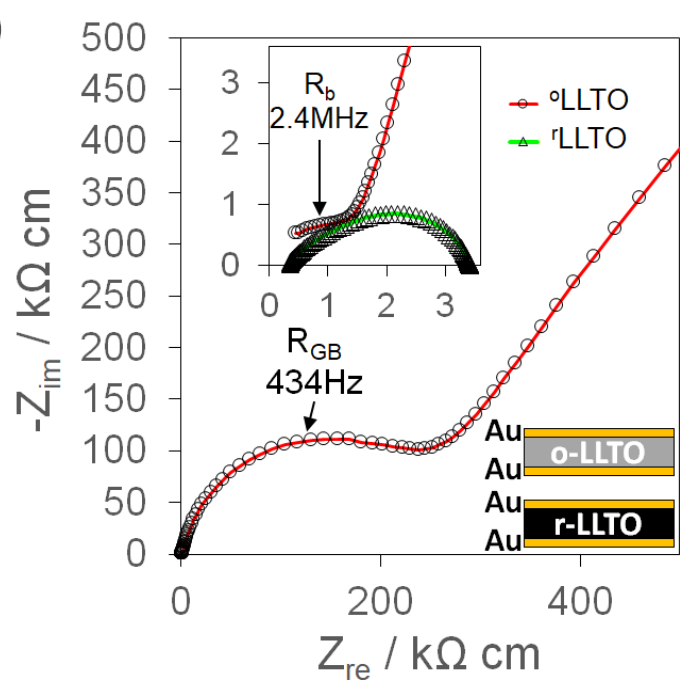

b)

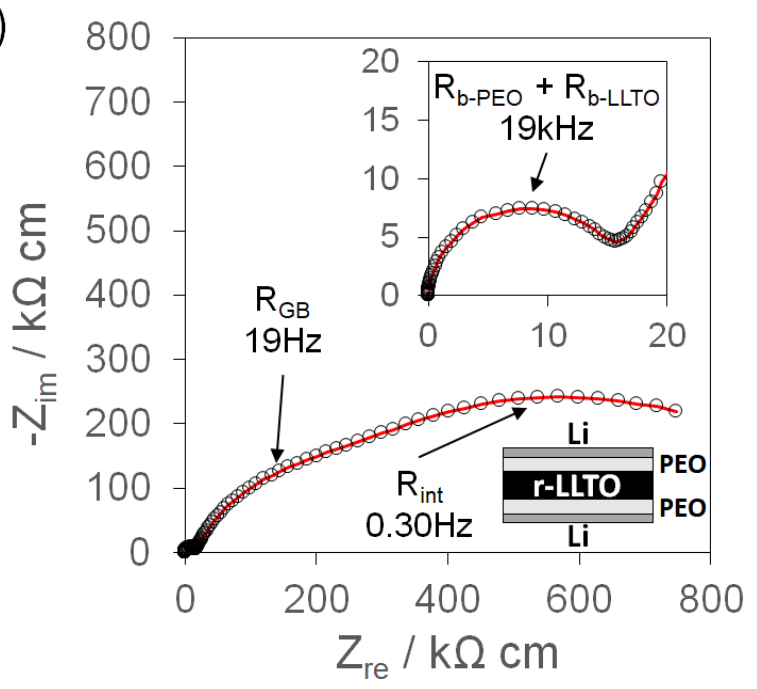

Figure 3. a) Schematic of EIS with Au Li-ion blocking electrodes and corresponding EIS for r-LLTO and o-LLTO at room temperature. b) schematic of EIS with electron blocking electrodes and corresponding EIS for r-LLTO at room temperature.

This article is protected by copyright. All rights reserved. 


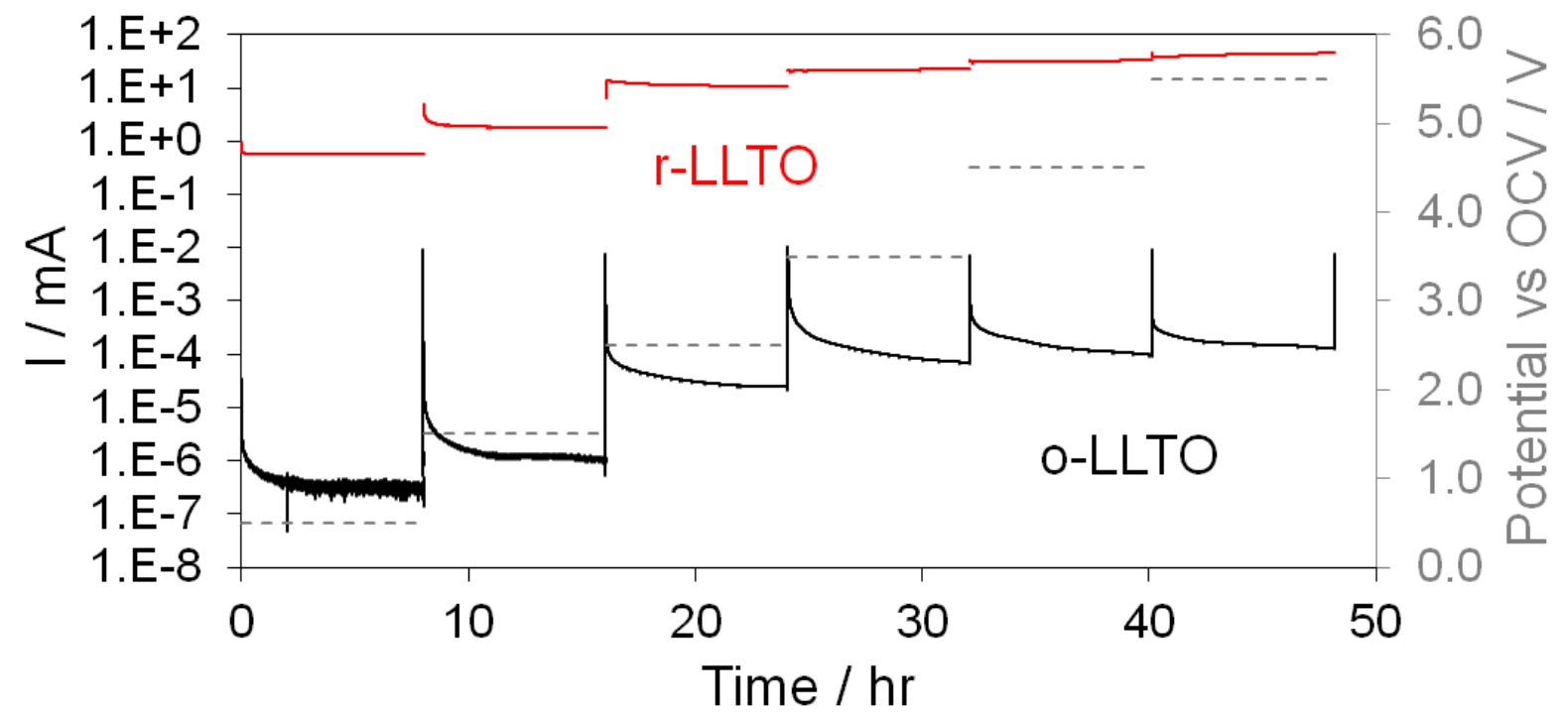

Figure 4. Chronoamperometry measurements for r-LLTO and o-LLTO.

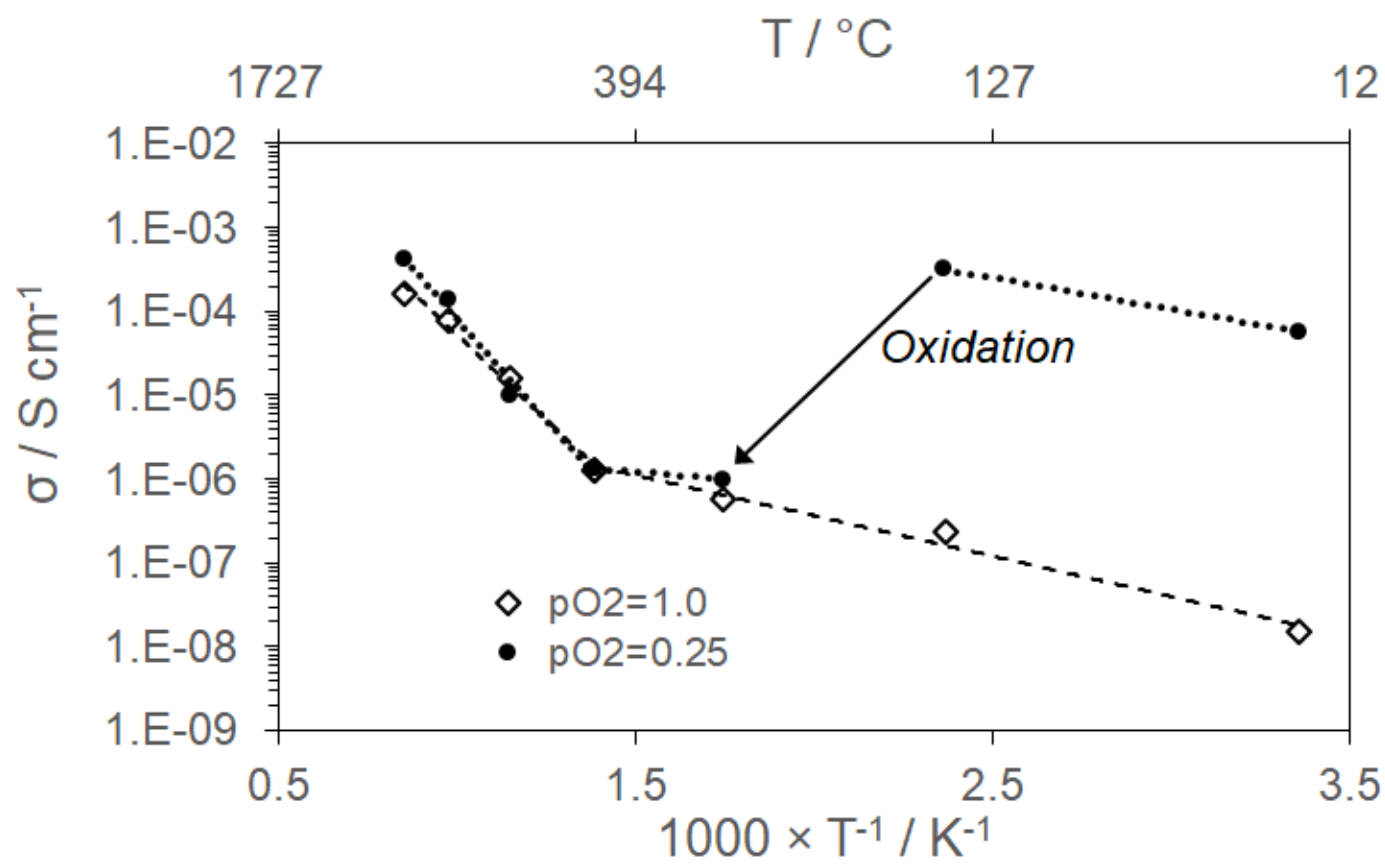

Figure 5. Arrhenius behavior of the electronic conductivity of LLTO measured in a $p_{O 2}=1.0$ and a $p_{O 2}=0.25$ atmosphere.

This article is protected by copyright. All rights reserved. 


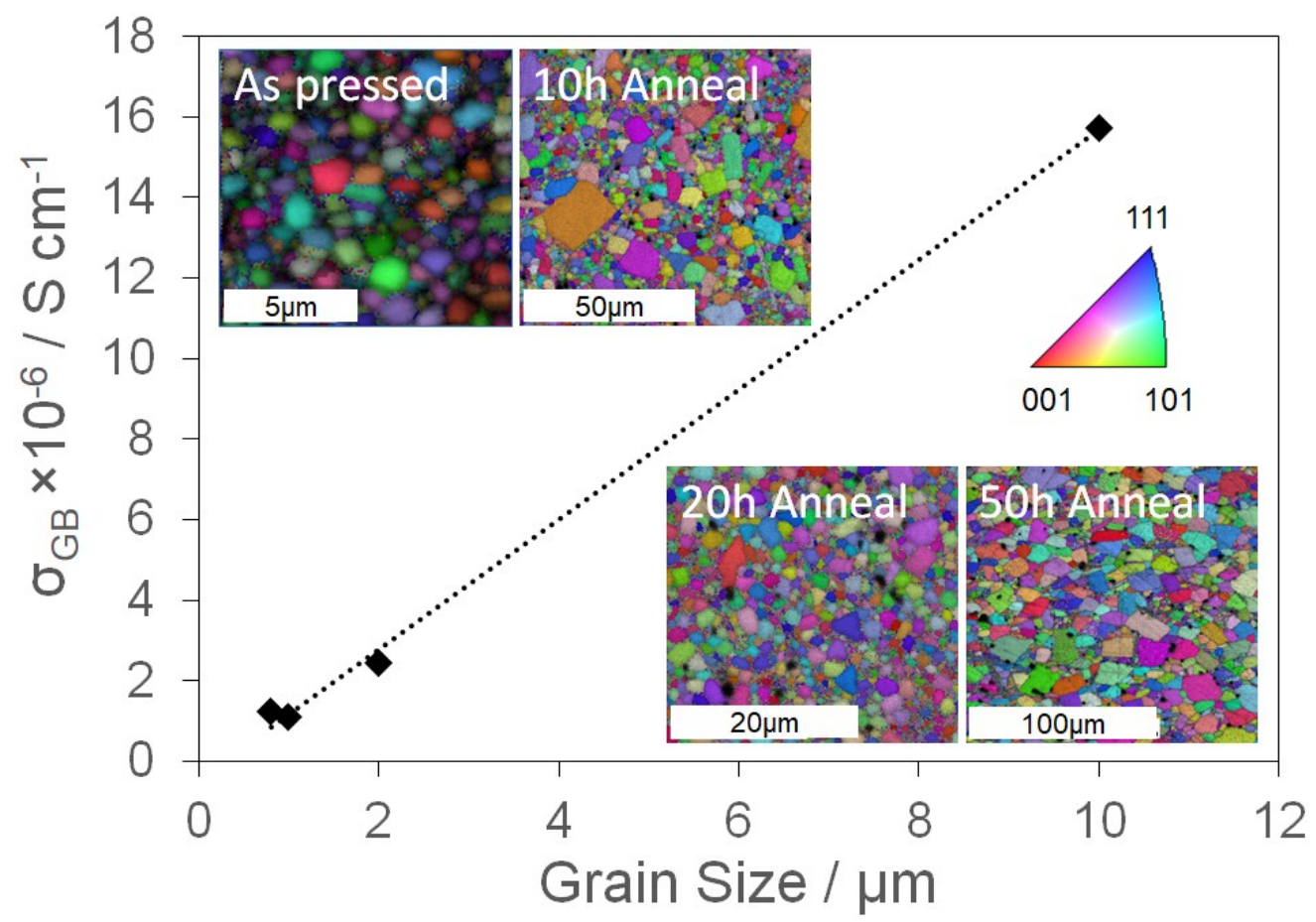

Figure 6. EBSD maps for as hot-pressed $r$-LLTO as well as $r$-LLTO annealed at $1150^{\circ} \mathrm{C}$ in $\mathrm{Ar}$ for $10-50$ hrs. Ionic grain boundary conductivity is shown as a function of average grain size.

This article is protected by copyright. All rights reserved. 
Lithium lanthanum titanate can exhibit both high ionic conductivity and high electronic conductivity depending on its oxidation state. While electronic conductivity in solid electrolytes is typically undesirable, the ability to conduct both Li-ions and electrons may pose useful for cathode composites. This work investigates the conduction properties of lithium lanthanum titanate and potential methods to control transference number.

Keyword lithium lanthanum titanate

M. Wang J. Wolfenstine and J. Sakamoto*

Mixed Electronic and lonic Conduction Properties of Lithium Lanthanum Titanate

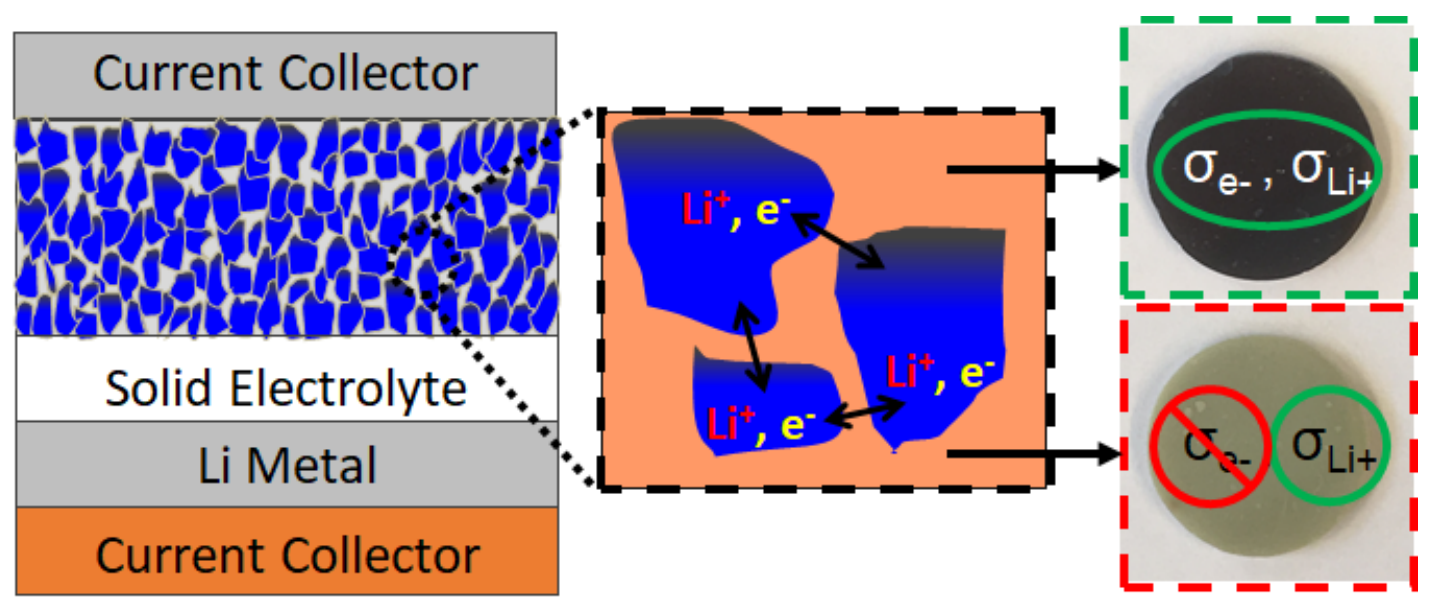

This article is protected by copyright. All rights reserved. 